MATHEMATICS OF COMPUTATION

Volume 71, Number 238, Pages 649-668

S $0025-5718(01) 01347-3$

Article electronically published on November 9, 2001

\title{
LANDEN TRANSFORMATIONS AND THE INTEGRATION OF RATIONAL FUNCTIONS
}

\author{
GEORGE BOROS AND VICTOR H. MOLL
}

\begin{abstract}
We present a rational version of the classical Landen transformation for elliptic integrals. This is employed to obtain explicit closed-form expressions for a large class of integrals of even rational functions and to develop an algorithm for numerical integration of these functions.
\end{abstract}

\section{INTRODUCTION}

We consider the space of even rational functions of degree $2 p$

$$
\mathfrak{E}_{2 p}:=\left\{R(z)=\frac{P(z)}{Q(z)} \mid P(z):=\sum_{k=0}^{p-1} b_{k} z^{2(p-1-k)} \text { and } Q(z):=\sum_{k=0}^{p} a_{k} z^{2(p-k)}\right\}
$$

with positive real coefficients $a_{k}, b_{k} \in \mathbb{R}_{+}$normalized by the condition $a_{0}=a_{p}=1$, the space

$$
\mathfrak{E}_{\infty}:=\bigcup_{p=1}^{\infty} \mathfrak{E}_{2 p}
$$

of normalized even rational functions, and the set of $2 p-1$ parameters

$$
\mathfrak{P}_{2 p}:=\left\{a_{1}, \cdots, a_{p-1} ; b_{0}, \cdots, b_{p-1}\right\} .
$$

We describe an algorithm to determine, as a function of the parameter set $\mathfrak{P}_{2 p}$, a closed-form expression of the integral

$$
I \quad:=\int_{0}^{\infty} R(z) d z
$$

for a large class of functions $R \in \mathfrak{E}_{\infty}$. The function $R$ is called symmetric if its denominator $Q$ satisfies $Q(1 / z)=z^{-2 p} Q(z)$. This is equivalent to its coefficients being palindromic, i.e., $a_{j}=a_{p-j}$ for $1 \leq j \leq p$.

The class of symmetric functions plays a crucial role in this algorithm. Define

$$
\mathfrak{E}_{2 p}^{s}:=\left\{R \in \mathfrak{E}_{2 p} \mid \operatorname{den}(R) \text { is symmetric }\right\}
$$

Received by the editor April 27, 1999.

2000 Mathematics Subject Classification. Primary 33-XX.

Key words and phrases. Rational functions, Landen transformation, integrals.

The second author was supported in part by NSF Grant DMS-0070567.

(C)2001 American Mathematical Society 
(where $\operatorname{den}(R)$ denotes the denominator of $R$ ), the class of rational functions with symmetric denominators of degree $2 p$, and

$$
\mathfrak{E}_{\infty}^{s}:=\bigcup_{p=1}^{\infty} \mathfrak{E}_{2 p}^{s}
$$

For $m \in \mathbb{N}$ define

$$
\mathfrak{E}_{2 p}^{m}:=\left\{R \in \mathfrak{E}_{\infty} \mid(\operatorname{den}(R))^{1 /(m+1)} \text { is even, symmetric of degree } 2 p\right\}
$$

and $\mathfrak{E}_{2 p}^{m, s}:=\mathfrak{E}_{2 p}^{m} \cap \mathfrak{E}_{2 p}^{s}$, so a function $R \in \mathfrak{E}_{2 p}^{m, s}$ can be written in the form

$$
R(z)=\frac{P(z)}{Q^{m+1}(z)}
$$

where $P(z)$ is an even polynomial and $Q(z)$ is an even symmetric polynomial of degree $2 p$.

The method of partial fractions gives (in principle) the value of $I$ in terms of the roots of $Q$. Symbolic computations yield either a closed-form answer, an expression in terms of the roots of an associated polynomial, or the integral returned unevaluated.

The algorithm described here allows only algebraic operations on elementary functions and changes of variables of the same type. In particular, we exclude the solution of algebraic equations of degree higher than 2. We say that a rational function $R \in \mathfrak{E}_{\infty}$ is computable if its integral can be evaluated by our algorithm.

The first step in the algorithm is to consider symmetric rational functions. In Section 2 we prove a reduction formula, i.e., a map $\mathfrak{F}_{p}: \mathfrak{E}_{2 p}^{s} \rightarrow \mathfrak{R}_{p}$ that reduces the computability of the integral of the symmetric function $R$ to that of one of degree $\frac{1}{2} \operatorname{deg}(R)$. Here $\mathfrak{R}_{p}$ is the space of rational functions with denominator of degree $p$. These new functions are not necessarily symmetric, and this is the main limitation of our algorithm. The details of $\mathfrak{F}_{p}$ require the evaluation of some binomial sums which are presented in Appendix A. The classical Wallis' formula

$$
\int_{0}^{\infty} \frac{d z}{\left(z^{2}+1\right)^{m+1}}=\frac{\pi}{2^{2 m+1}}\left(\begin{array}{c}
2 m \\
m
\end{array}\right)
$$

shows that every $R \in \mathfrak{E}_{2}^{m}$ is computable. The reduction formula now implies that every $R \in \mathfrak{E}_{4}^{m}$ is computable. This is described in Section 3. The computability of $R \in \mathfrak{E}_{4}$ is also a consequence of the classical theory of hypergeometric functions; the details are given in [2]. In Section 4 we compute the integral of every function in $\mathfrak{E}_{8}^{m, s}$, where the reduction method expresses these integrals in terms of functions in $\mathfrak{E}_{4}^{m}$. The algorithm does not, in general, provide a value for the integral of a nonsymmetric function of degree 8 .

The final piece of the algorithm is described in Section 5: for $R \in \mathfrak{E}_{2 p}$, the symmetrization of its denominator produces a (symmetric) rational function in $\mathfrak{E}_{4 p}$ with the same integral as $R$. The reduction formula now yields a new function in $\mathfrak{E}_{2 p}$ with the same integral as $R$. We thus obtain a map $\mathfrak{T}_{2 p}: \mathfrak{E}_{2 p} \rightarrow \mathfrak{E}_{2 p}$ such that

$$
\int_{0}^{\infty} R(z) d z=\int_{0}^{\infty} \mathfrak{T}_{2 p}(R(z)) d z .
$$

In particular, the class of computable rational functions of degree $2 p$ is invariant under forward and backward iteration of $\mathfrak{T}_{2 p}$. This map is the rational analog of the original Landen transformation for elliptic integrals described in [4, 13]. The 
map $\mathfrak{T}_{2 p}$ can also be interpreted as a map on the coefficients $\Phi_{2 p}: \mathfrak{O}_{2 p}^{+} \rightarrow \mathfrak{O}_{2 p}^{+}$ where $\mathfrak{O}_{2 p}^{+}=\mathbb{R}_{+}{ }^{p-1} \times \mathbb{R}_{+}{ }^{p}$.

The case of $\Phi_{6}$ is described in detail in Section [6 and is given explicitly by

$$
\begin{aligned}
a_{1} & \rightarrow \frac{9+5 a_{1}+5 a_{2}+a_{1} a_{2}}{\left(a_{1}+a_{2}+2\right)^{4 / 3}}, \\
a_{2} & \rightarrow \frac{a_{1}+a_{2}+6}{\left(a_{1}+a_{2}+2\right)^{2 / 3}} \\
b_{0} & \rightarrow \frac{b_{0}+b_{1}+b_{2}}{\left(a_{1}+a_{2}+2\right)^{2 / 3}} \\
b_{1} & \rightarrow \frac{b_{0}\left(a_{2}+2\right)+2 b_{1}+b_{2}\left(a_{1}+3\right)}{a_{1}+a_{2}+2} \\
b_{2} & \rightarrow \frac{b_{0}+b_{2}}{\left(a_{1}+a_{2}+2\right)^{1 / 3}}
\end{aligned}
$$

Let $\mathbf{x}_{0}:=\left(a_{1}, a_{2} ; b_{0}, b_{1}, b_{2}\right)$. Then $\Phi_{6}: \mathfrak{O}_{6}^{+} \rightarrow \mathfrak{O}_{6}^{+}$is iterated to produce a sequence $\mathbf{x}_{n+1}:=\Phi_{6}\left(\mathbf{x}_{n}\right)$ of points in $\mathfrak{O}_{6}^{+}$that yield a sequence of rational functions with constant integral. We have proved in [3] the existence of $L \in \mathbb{R}^{+}$, depending upon the initial point $\mathbf{x}_{0}=\left(a_{1}, a_{2} ; b_{0}, b_{1}, b_{2}\right)$, such that $\mathbf{x}_{n} \rightarrow(3,3 ; L, 2 L, L)$. Thus

$$
\int_{0}^{\infty} \frac{b_{0} z^{4}+b_{1} z^{2}+b_{2}}{z^{6}+a_{1} z^{4}+a_{2} z^{2}+1} d z=L\left(\mathbf{x}_{0}\right) \times \frac{\pi}{2} .
$$

This establishes a numerical method to compute the integral in (1.4).

Numerical studies on integrals of even degree $2 p$ suggest the existence of a limiting value $L=L\left(\mathbf{x}_{0}\right)$ such that the sequence $\mathbf{x}_{n+1}:=\Phi_{2 p}\left(\mathbf{x}_{n}\right)$ satisfies

$$
\mathbf{x}_{n} \rightarrow\left(\left(\begin{array}{l}
p \\
1
\end{array}\right),\left(\begin{array}{l}
p \\
2
\end{array}\right), \cdots,\left(\begin{array}{c}
p \\
p-1
\end{array}\right) ;\left(\begin{array}{c}
p-1 \\
0
\end{array}\right) L,\left(\begin{array}{c}
p-1 \\
1
\end{array}\right) L, \cdots,\left(\begin{array}{c}
p-1 \\
p-1
\end{array}\right) L\right) .
$$

The integral of the original rational function is thus $\frac{\pi}{2} \times L\left(\mathbf{x}_{0}\right)$. The proof of convergence remains open for $p \geq 4$. Examples are given in Section 7 .

The most important issues left unresolved in this paper are the convergence of the iteration of the map $\Phi_{2 p}$ discussed above and the geometric interpretation of the Landen transformation $\mathfrak{T}_{2 p}$. Finally, the question of integration of odd functions has not been addressed at all.

Some history. The problem of integration of rational functions $R(z)=P(z) / Q(z)$ was considered by J. Bernoulli in the 18th century. He completed the original attempt by Leibniz of a general partial fraction decomposition of $R(z)$. The main difficulty associated with this procedure is to obtain a complete factorization of $Q(z)$ over $\mathbb{R}$. Once this is known the partial fraction decomposition of $R(z)$ can be computed. The fact is that the primitive of a rational function is always elementary: it consists of a new rational function (its rational part) and the logarithm of a second rational function (its transcendental part). In his classic monograph 9] G. H. Hardy states: The solution of the problem (of definite integration) in the case of rational functions may therefore be said to be complete; for the difficulty with regard to the explicit solution of algebraical equations is one not of inadequate knowledge but of proved impossibility. He goes on to add: It appears from the preceding paragraphs that we can always find the rational part of the integral, and can find the complete integral if we can find the roots of $Q(z)=0$. 
In the middle of the last century Hermite [10] and Ostrogradsky 15] developed algorithms to compute the rational part of the primitive of $R(z)$ without factoring $Q(z)$. More recently Horowitz [1] rediscovered this method and discussed its complexity. The problem of computing the transcendental part of the primitive was finally solved by Lazard and Rioboo [12], Rothstein 17] and Trager 18]. For detailed descriptions and proofs of these algorithms the reader is referred to [5] and [6].

\section{The REDUCTION FORMula}

In this section we present a map $\mathfrak{F}_{p}: \mathfrak{E}_{2 p}^{m, s} \rightarrow \mathfrak{E}_{p}^{m}$ that is the basis of the integration algorithm described in Section [5] The proof is elementary and the binomial sums discussed in the Appendix are employed.

Let $D_{p}(z)$ be the general symmetric polynomial of degree $4 p$. We express the integral of $z^{2 n} / D_{p}^{m+1}$ as a linear combination of integrals of $z^{2 j} / E_{p}^{m+1}$ where $E_{p}$ is a polynomial of degree $2 p$ whose coefficients are determined by those of $D_{p}$.

Theorem 2.1. Let $m, n, p \in \mathbb{N}$. Define

$$
D_{p}\left(d_{1}, d_{2}, \cdots, d_{p} ; z\right)=\sum_{k=0}^{p} d_{p+1-k}\left(z^{2 k}+z^{4 p-2 k}\right)
$$

and

$$
\begin{aligned}
E_{p}\left(d_{1}, d_{2}, \cdots, d_{p} ; z\right)= & \left(\sum_{j=1}^{p+1} d_{j}\right) z^{2 p} \\
& +\sum_{i=1}^{p} 2^{2 i-1} z^{2(p-i)} \sum_{j=1}^{p-i+1} \frac{j+i-1}{i}\left(\begin{array}{c}
j+2 i-2 \\
j-1
\end{array}\right) d_{j+i},
\end{aligned}
$$

for $d_{i} \in \mathbb{R}_{+}$. Then for $0 \leq n \leq(m+1) p-1$,

$$
\begin{aligned}
\int_{0}^{\infty} & \frac{z^{2 n} d z}{\left(D_{p}\left(d_{1}, \cdots, d_{p} ; z\right)\right)^{m+1}} \\
= & 2^{-m} \sum_{j=0}^{(m+1) p-n-1} 4^{j}\left(\begin{array}{c}
(m+1) p-n-1+j \\
2 j
\end{array}\right) \\
& \times \int_{0}^{\infty} \frac{z^{2((m+1) p-1-j)}}{\left(E_{p}\left(d_{1}, \cdots, d_{p} ; z\right)\right)^{m+1}} d z
\end{aligned}
$$

and for $(m+1) p-1<n<2 p(m+1)-1$ we employ the symmetry rule

$$
N_{n, p}=N_{2 p(m+1)-1-n, p}
$$

Proof. First observe that (2.4) follows from the change of variable $z \rightarrow 1 / z$. Now consider

$$
N_{n, p}\left(d_{1}, \cdots, d_{p} ; m\right):=\int_{0}^{\infty} \frac{z^{2 n} d z}{\left(\sum_{k=0}^{p} d_{p+1-k}\left(z^{4 p-2 k}+z^{2 k}\right)\right)^{m+1}}
$$


for $0 \leq n \leq(m+1) p-1$. The substitution $z=\tan \theta$ yields

$$
N_{n, p}=\int_{0}^{\pi / 2} \frac{\left(1-C^{2}\right)^{n} C^{4(m+1) p-2 n-2} d \theta}{\left(\sum_{k=0}^{p} d_{p+1-k}\left\{\left(1-C^{2}\right)^{2 p-k} C^{2 k}+\left(1-C^{2}\right)^{k} C^{4 p-2 k}\right\}\right)^{m+1}},
$$

where $C=\cos \theta$. Letting $\psi=2 \theta$ and $D=\cos \psi=2 C^{2}-1$ then gives

$$
N_{n, p}=\int_{0}^{\pi} \frac{\left(1-D^{2}\right)^{n}(1+D)^{2(m+1) p-2 n-1} d \psi}{\left(\sum_{k=0}^{p} d_{p+1-k}\left(1-D^{2}\right)^{k}\left\{(1-D)^{2 p-2 k}+(1+D)^{2 p-2 k}\right\}\right)^{m+1}} .
$$

Now observe that the integrals of the odd powers of cosine vanish when we expand $(1+D)^{2(m+1) p-2 n-1}$, producing

$$
N_{n, p}=2^{-m} \int_{0}^{\pi / 2} \frac{\left(1-D^{2}\right)^{n} \sum_{j=0}^{(m+1) p-n-1}\left(\begin{array}{c}
2(m+1) p-2 n-1 \\
2 j
\end{array}\right) D^{2 j} d \theta}{\left\{\sum_{k=0}^{p} d_{p+1-k}\left(1-D^{2}\right)^{k} \sum_{j=0}^{p-k}\left(\begin{array}{c}
2 p-2 k \\
2 j
\end{array}\right) D^{2 j}\right\}^{m+1}} .
$$

A second double angle substitution $\varphi=2 \psi$ gives

$$
N_{n, p}=2^{-m} \int_{0}^{\pi} \frac{(1-E)^{n} \sum_{j=0}^{(m+1) p-n-1}\left(\begin{array}{c}
(m+1) p-2 n-1 \\
2 j
\end{array}\right) 2^{p(m+1)-n-j-1}(1+E)^{j} d \varphi}{\left\{\sum_{k=0}^{p} d_{p+1-k}(1-E)^{k} \sum_{j=0}^{p-k}\left(\begin{array}{c}
2 p-2 k \\
2 j
\end{array}\right) 2^{p-k-j}(1+E)^{j}\right\}^{m+1}},
$$

where $E=\cos \varphi=2 D^{2}-1$. The change of variable $z=\tan (\varphi / 2)$ then yields

$$
N_{n, p}=2^{-m} \int_{0}^{\infty} \frac{z^{2 n} \sum_{j=0}^{(m+1) p-n-1}\left(\begin{array}{c}
2(m+1) p-2 n-1 \\
2 j
\end{array}\right)\left(1+z^{2}\right)^{(m+1) p-n-j-1} d z}{\left\{\sum_{k=0}^{p} d_{p+1-k} z^{2 k}\left(\sum_{j=0}^{p-k}\left(\begin{array}{c}
2 p-2 k \\
2 j
\end{array}\right)\left(1+z^{2}\right)^{p-k-j}\right)\right\}^{m+1}} .
$$

Finally, we modify (2.6) using Lemma A.2 and Lemma A.4 with $N=(m+1) p-n-1$ to produce (2.3).

Note that the previous theorem associates to each rational function of symmetric denominator

$$
R_{1}(z)=\frac{b_{s} z^{2 s}+b_{s-1} z^{2(s-1)}+\cdots+b_{0}}{\left(z^{4 p}+d_{p} z^{4 p-2}+\cdots+2 d_{1} z^{2 p}+\cdots+1\right)^{m+1}}
$$

a new rational function

$$
\begin{aligned}
R_{2}(z)= & 2^{-m} \sum_{n=0}^{(m+1) p-1} b_{n} \sum_{j=0}^{(m+1) p-n-1} 4^{j} \\
& \times\left(\begin{array}{c}
(m+1) p-n-1+j \\
2 j
\end{array}\right) \frac{z^{2((m+1) p-1-j)}}{\left(E_{p}\left(d_{1}, \cdots, d_{p} ; z\right)\right)^{m+1}}
\end{aligned}
$$

such that

$$
\int_{0}^{\infty} R_{1}(z) d z=\int_{0}^{\infty} R_{2}(z) d z
$$




\section{The QuARTIC CASE}

In this section we describe the computability of rational functions $R \in \mathfrak{E}_{4}^{m}$. These are functions of the form

$$
R(z)=\frac{P(z)}{\left(z^{4}+2 a z^{2}+1\right)^{m+1}},
$$

where $P(z)$ is an even polynomial of degree $4 m+2$. Observe that the normalization $a_{0}=a_{2}=1$ makes the denominator of $R$ automatically symmetric. It suffices to evaluate

$$
N_{n, 4}\left(d_{1} ; m\right):=\int_{0}^{\infty} \frac{z^{2 n} d z}{\left(z^{4}+2 d_{1} z^{2}+1\right)^{m+1}},
$$

where $0 \leq n \leq 2 m+1$ is required for convergence. From (2.4) we have $N_{n, 4}\left(d_{1} ; m\right)=$ $N_{2 m-1-n, 4}\left(d_{1} ; m\right)$, so we may assume $0 \leq n \leq m$. We now employ Theorem [2.1] to obtain a closed form expression for $N_{n, 4}\left(d_{1} ; m\right)$.

Theorem 3.1. Let $m \in \mathbb{N}$ and assume $0 \leq n \leq m$. Then

$$
\begin{aligned}
N_{n, 4}\left(d_{1} ; m\right):= & \int_{0}^{\infty} \frac{z^{2 n} d z}{\left(z^{4}+2 d_{1} z^{2}+1\right)^{m+1}} \\
= & \frac{\pi}{2^{3 m+3 / 2}(1+a)^{m+1 / 2}} \times \sum_{j=0}^{m-n} 2^{j}\left(1+d_{1}\right)^{j} \\
& \times\left(\begin{array}{c}
2 m-2 j-1 \\
m-j
\end{array}\right)\left(\begin{array}{c}
m-n+j \\
2 j
\end{array}\right)\left(\begin{array}{c}
2 j \\
j
\end{array}\right)\left(\begin{array}{c}
m \\
j
\end{array}\right)^{-1} .
\end{aligned}
$$

For $m+1 \leq n \leq 2 m+1$ we have

$$
\begin{aligned}
\int_{0}^{\infty} & \frac{z^{2 n} d z}{\left(z^{4}+2 d_{1} z^{2}+1\right)^{m+1}} \\
= & \frac{\pi}{2^{3 m+3 / 2}\left(1+d_{1}\right)^{m+1 / 2}} \\
& \times \sum_{j=0}^{n-m-1} 2^{j}\left(1+d_{1}\right)^{j} \times\left(\begin{array}{c}
2 m-2 j-1 \\
m-j
\end{array}\right)\left(\begin{array}{c}
m-n+j \\
2 j
\end{array}\right)\left(\begin{array}{c}
2 j \\
j
\end{array}\right)\left(\begin{array}{c}
m \\
j
\end{array}\right)^{-1} .
\end{aligned}
$$

Proof. We apply the result of the Theorem 2.1 with $D_{1}\left(d_{1} ; z\right)=z^{4}+2 d_{1} z^{2}+1$ and $E_{1}\left(d_{1} ; z\right)=\left(1+d_{1}\right) z^{2}+2$, so that

$$
\int_{0}^{\infty} \frac{z^{2 n} d z}{\left(z^{4}+2 d_{1} z^{2}+1\right)^{m+1}}=2^{-m} \sum_{j=0}^{m-n} 4^{j}\left(\begin{array}{c}
m-n+j \\
2 j
\end{array}\right) \int_{0}^{\infty} \frac{z^{2(m-j)} d z}{\left(\left(1+d_{1}\right) z^{2}+2\right)^{m+1}} .
$$

The change of variable $u=\left(1+d_{1}\right) z^{2} / 2$ then yields

$$
\begin{aligned}
\int_{0}^{\infty} & \frac{z^{2(m-j)} d z}{\left(\left(1+d_{1}\right) z^{2}+2\right)^{m+1}} \\
& =2^{-(j+3 / 2)}\left(1+d_{1}\right)^{-m+j-1 / 2} \int_{0}^{\infty} \frac{u^{m-j-1 / 2} d u}{(1+u)^{m+1}} \\
& =\pi\left(\begin{array}{c}
2 m-2 j \\
m-j
\end{array}\right)\left(\begin{array}{c}
2 j \\
j
\end{array}\right)\left(\begin{array}{c}
m \\
j
\end{array}\right)^{-1} 2^{-(2 m+j+3 / 2)}\left(1+d_{1}\right)^{-(m-j+1 / 2)},
\end{aligned}
$$


where we have used

$$
\int_{0}^{\infty} \frac{u^{r-1 / 2}}{(1+u)^{s}} d u=\frac{\pi}{2^{2(s-1)}}\left(\begin{array}{c}
2 r \\
r
\end{array}\right)\left(\begin{array}{c}
2(s-r-1) \\
s-r-1
\end{array}\right)\left(\begin{array}{c}
s-1 \\
r
\end{array}\right)^{-1} .
$$

The algorithm also requires a scaled version of $N_{0,4}\left(d_{1} ; m\right)$.

Corollary 3.2. Let $b>0, c>0, a>-\sqrt{b c}, m \in \mathbb{N}$, and $0 \leq n \leq m$. Define

$$
N_{n, 4}(a, b, c ; m):=\int_{0}^{\infty} \frac{z^{2 n} d z}{\left(b z^{4}+2 a z^{2}+c\right)^{m+1}} .
$$

Then for $0 \leq n \leq m$,

$$
\begin{aligned}
N_{n, 4}(a, b, c ; m) \\
=\pi\left(c(c / b)^{m-n}\{8(a+\sqrt{b c})\}^{2 m+1}\right)^{-1 / 2} \\
\quad \times \sum_{k=0}^{m-n} 2^{k}\left(\begin{array}{c}
2 m-2 k \\
m-k
\end{array}\right)\left(\begin{array}{c}
m-n+k \\
2 k
\end{array}\right)\left(\begin{array}{c}
2 k \\
k
\end{array}\right)\left(\begin{array}{c}
m \\
k
\end{array}\right)^{-1}\left(\frac{a}{\sqrt{b c}}+1\right)^{k},
\end{aligned}
$$

and for $m+1 \leq n \leq 2 m+1$,

$$
\begin{aligned}
N_{n, 4}(a, b, c ; m) \\
=\pi\left(c(c / b)^{m-n}\{8(a+\sqrt{b c})\}^{2 m+1}\right)^{-1 / 2} \\
\quad \times \sum_{k=0}^{n-m-1} 2^{k}\left(\begin{array}{c}
2 m-2 k \\
m-k
\end{array}\right)\left(\begin{array}{c}
m-n+k \\
2 k
\end{array}\right)\left(\begin{array}{c}
2 k \\
k
\end{array}\right)\left(\begin{array}{c}
m \\
k
\end{array}\right)^{-1}\left(\frac{a}{\sqrt{b c}}+1\right)^{k} .
\end{aligned}
$$

Proof. Let $0 \leq n \leq m$. The substitution $u=z(b / c)^{1 / 4}$ yields

$$
N_{n, 4}(a, b, c ; m)=\frac{1}{c^{m-n / 2+3 / 4} b^{n / 2+1 / 4}} N_{n, 4}\left(\frac{a}{\sqrt{b c}} ; m\right),
$$

so (3.4) then follows from Theorem 3.1 From (2.4) we have $N_{n, 4}(a, b, c ; m)=$ $N_{2 m+1-n, 4}(c, b, a ; m)$ for $m+1 \leq n \leq 2 m+1$, giving (3.5).

\section{The Symmetric CASE OF DEGREE 8}

In this section we prove the computability of the set $\mathfrak{E}_{8}^{m, s}$ of symmetric rational functions with denominator of degree 8 and establish an explicit formula for the integral

$$
N_{n, 8}\left(a_{1}, a_{2} ; m\right)=\int_{0}^{\infty} \frac{z^{2 n} d z}{\left(z^{8}+a_{2} z^{6}+2 a_{1} z^{4}+a_{2} z^{2}+1\right)^{m+1}}
$$

where $0 \leq n \leq 4 m+3$ is required for convergence. Observe that (2.4) reduces the discussion to the case $0 \leq n \leq 2 m+1$. The expression (2.2), with $p=2$, produces $E_{2}\left(a_{1}, a_{2} ; z\right)=\left(1+a_{1}+a_{2}\right) z^{4}+2\left(a_{2}+4\right) z^{2}+8$. 
Theorem 4.1. Every function in $\mathfrak{E}_{8}^{m, s}$ is computable. More specifically, define $c_{1}:=a_{2}+4, c_{2}:=1+a_{1}+a_{2}$, and

$$
\begin{aligned}
t_{k, j}\left(m, n ; a_{1}, a_{2}\right) & :=\pi 2^{-(3 m+2+k+j) / 2} c_{2}^{(m-k-j) / 2}\left(c_{1}+\sqrt{8 c_{2}}\right)^{j-m-1 / 2} \\
& \times\left(\begin{array}{c}
4 m-n-k+2 \\
k-n
\end{array}\right)\left(\begin{array}{c}
2 m-2 j \\
m-j
\end{array}\right)\left(\begin{array}{c}
m-k+j \\
2 j
\end{array}\right)\left(\begin{array}{c}
2 j \\
j
\end{array}\right)\left(\begin{array}{c}
m \\
j
\end{array}\right)^{-1} .
\end{aligned}
$$

Then for $m+1 \leq n \leq 2 m+1,1+a_{1}+a_{2}>0$ and $a_{2}+4>-8 \sqrt{8\left(1+a_{1}+a_{2}\right)}$,

$$
\int_{0}^{\infty} \frac{z^{2 n} d z}{\left(z^{8}+a_{2} z^{6}+2 a_{1} z^{4}+a_{2} z^{2}+1\right)^{m+1}}=\sum_{k=n}^{2 m+1} \sum_{j=0}^{k-m-1} t_{k, j}\left(m, n ; a_{1}, a_{2}\right),
$$

and for $0 \leq n \leq m$,

$$
\begin{aligned}
\int_{0}^{\infty} & \frac{z^{2 n} d z}{\left(z^{8}+a_{2} z^{6}+2 a_{1} z^{4}+a_{2} z^{2}+1\right)^{m+1}} \\
& =\sum_{k=n}^{m} \sum_{j=0}^{m-k} t_{k, j}\left(m, n ; a_{1}, a_{2}\right)+\sum_{k=m+1}^{2 m+1} \sum_{j=0}^{k-m-1} t_{k, j}\left(m, n ; a_{1}, a_{2}\right) .
\end{aligned}
$$

Proof. The reduction formula yields

$$
\begin{aligned}
\int_{0}^{\infty} & \frac{z^{2 n} d z}{\left(z^{8}+a_{2} z^{6}+2 a_{1} z^{4}+a_{2} z^{2}+1\right)^{m+1}} \\
& =2^{3 m+2} \sum_{k=n}^{2 m+1} 2^{-2 k}\left(\begin{array}{c}
4 m-n-k+2 \\
k-n
\end{array}\right) \int_{0}^{\infty} \frac{z^{2 k} d z}{\left(c_{2} z^{4}+2 c_{1} z^{2}+8\right)^{m+1}}
\end{aligned}
$$

We then use Corollary 3.2 to evaluate (4.1).

\section{A SEQUENCE of LANDEN TRANSFORMations}

The transformation theory of elliptic integrals was initiated by Landen in 1771 . He proved the invariance of the function

$$
G(a, b):=\int_{0}^{\pi / 2} \frac{d \theta}{\sqrt{a^{2} \cos ^{2} \theta+b^{2} \sin ^{2} \theta}}
$$

under the transformation

$$
a_{1}=(a+b) / 2, \quad b_{1}=\sqrt{a b},
$$

i.e., that

$$
G\left(a_{1}, b_{1}\right)=G(a, b) .
$$

Gauss [7] rediscovered this invariance while numerically calculating the length of a lemniscate. An elegant proof of (5.3) is given by Newman in [14. Here, the substitution $x=b \tan \theta$ converts $2 G(a, b)$ into the integral of $\left[\left(a^{2}+x^{2}\right)\left(b^{2}+x^{2}\right)\right]^{-1 / 2}$ over $\mathbb{R}$; the change of variable $t=(x-a b / x) / 2$ then completes the proof.

The Gauss-Landen transformation can be iterated to produce a double sequence $\left(a_{n}, b_{n}\right)$ such that $0 \leq a_{n}-b_{n}<2^{-n}$. It follows that $a_{n}$ and $b_{n}$ converge to a common 
limit, the so-called arithmetic-geometric mean of $a$ and $b$, denoted by $\operatorname{AGM}(a, b)$. Passing to the limit in $G(a, b)=G\left(a_{n}, b_{n}\right)$ produces

$$
\frac{\pi}{2 \operatorname{AGM}(a, b)}=\int_{0}^{\pi / 2} \frac{d \theta}{\sqrt{a^{2} \cos ^{2} \theta+b^{2} \sin ^{2} \theta}}
$$

The reader is referred to [4] and [13] for details.

The goal of this section is to produce a map $\mathfrak{T}_{2 p}: \mathfrak{E}_{2 p} \rightarrow \mathfrak{E}_{2 p}$ that preserves the integral, i.e.,

$$
\int_{0}^{\infty} R(z) d z=\int_{0}^{\infty} \mathfrak{T}_{2 p}(R(z)) d z .
$$

This map is the rational analog of the original Landen transformation (5.2).

Theorem 5.1. Let $R(z)=P(z) / Q(z)$ with

$$
P(z)=\sum_{j=0}^{p-1} b_{j} z^{2(p-1-j)} \text { and } Q(z)=\sum_{j=0}^{p} a_{j} z^{2(p-j)} .
$$

Define $a_{j}=0$ for $j>p, b_{j}=0$ for $j>p-1$,

$$
d_{p+1-j}=\sum_{k=0}^{j} a_{p-k} a_{j-k}
$$

for $0 \leq k \leq p-1$,

$$
\begin{gathered}
d_{1}=\frac{1}{2} \sum_{k=0}^{p} a_{p-k}^{2}, \\
c_{j}=\sum_{k=0}^{2 p-1} a_{j} b_{p-1-j+k}
\end{gathered}
$$

for $0 \leq j \leq 2 p-1$, and also

$$
\alpha_{p}(i)=\left\{\begin{array}{l}
2^{2 i-1} \sum_{k=1}^{p+1-i} \frac{k+i-1}{i}\left(\begin{array}{c}
k+2 i-2 \\
k-1
\end{array}\right) d_{k+i} \quad \text { if } 1 \leq i \leq p, \\
1+\sum_{k=1}^{p} d_{k} \quad \text { if } i=0 .
\end{array}\right.
$$

Let

$$
a_{i}^{+}=\frac{\alpha_{p}(i)}{2^{2 i} Q(1)^{2(1-i / p)}}
$$

for $1 \leq i \leq p-1$, and

$$
b_{i}^{+}=Q(1)^{2 i / p+1 / p-2} \times\left[\sum_{k=0}^{p-1-i}\left(c_{k}+c_{2 p-1-k}\right)\left(\begin{array}{c}
p-1-k+i \\
2 i
\end{array}\right)\right]
$$

for $0 \leq i \leq p-1$. Finally, define the polynomials

$$
P^{+}(z)=\sum_{k=0}^{p-1} b_{i}^{+} z^{2(p-1-i)} \quad \text { and } \quad Q^{+}(z)=\sum_{k=0}^{p} a_{i}^{+} z^{2(p-i)} .
$$

Then $\mathfrak{T}_{2 p}(R(z)):=P^{+}(z) / Q^{+}(z)$ satisfies (5.5)), i.e.,

$$
\int_{0}^{\infty} \frac{P(z)}{Q(z)} d z=\int_{0}^{\infty} \frac{P^{+}(z)}{Q^{+}(z)} d z .
$$


Proof. The first step is to convert the polynomial $Q(z)$ to its symmetric form:

$$
I:=\int_{0}^{\infty} \frac{P(z)}{Q(z)} d z=\int_{0}^{\infty} \frac{C(z)}{D(z)} d z
$$

with

$$
\begin{aligned}
& C(z)=P(z) \times z^{2 p} Q(1 / z):=\sum_{k=0}^{2 p-1} c_{k} z^{2 k} \\
& D(z)=Q(z) \times z^{2 p} Q(1 / z):=\sum_{k=0}^{p} d_{p+1-k}\left(z^{2 k}+z^{2(2 p-k)}\right) .
\end{aligned}
$$

Then

$$
I=\sum_{k=0}^{2 p-1} c_{k} \int_{0}^{\infty} \frac{z^{2 k} d z}{Q^{s}(z)} .
$$

Now employ the reduction formula in Section 2 to evaluate

$$
L_{k}:=\int_{0}^{\infty} \frac{z^{2 k} d z}{Q^{s}(z)}
$$

Observe that one needs to evaluate $L_{k}$ only for $0 \leq k \leq p-1$. Indeed, the usual symmetry rule yields $L_{k}=L_{2 p-1-k}$. The reduction formula now gives

$$
\begin{aligned}
L_{k} & =\sum_{j=0}^{p-1-k} 2^{2 j}\left(\begin{array}{c}
p-1-k+j \\
2 j
\end{array}\right) \int_{0}^{\infty} \frac{z^{2(p-1-j)} d z}{\sum_{i=0}^{p} \alpha_{p}(i) z^{2(p-i)}} \\
& =\frac{1}{\alpha_{p}(p)} \sum_{j=1}^{p-k} 2^{2(j-1)}\left(\begin{array}{c}
p-k+j-2 \\
2 j-2
\end{array}\right) \lambda^{2 p-2 j+1} \int_{0}^{\infty} \frac{z^{2(p-j)} d z}{\sum_{i=0}^{p} b_{i}^{+} z^{2(p-i)}}
\end{aligned}
$$

with $\alpha_{p}(i)$ as in (5.10) and $\lambda=\left[\alpha_{p}(p) / \alpha_{p}(0)\right]^{1 / 2 p}$.

Note. The extension of this transformation to the case of

$$
\int_{0}^{\infty} \frac{P(z)}{Q^{m+1}(z)} d z
$$

requires explicit formulae for the coefficients of $P(z) \times\left(z^{2 p} Q(1 / z)\right)^{m+1}$ and $Q^{m+1}(z) \times\left(z^{2 p} Q(1 / z)\right)^{m+1}$.

An algorithm for integration. Let $\mathbf{x}=(\mathbf{a}, \mathbf{b})$ with $\mathbf{a}=\left(a_{1}, \cdots, a_{p-1}\right), \mathbf{b}=$ $\left(b_{0}, \cdots, b_{p-1}\right)$, and let $\mathfrak{O}_{2 p}^{+}=\mathbb{R}_{+}{ }^{p-1} \times \mathbb{R}_{+}{ }^{p}$. We then have a map

$$
\begin{aligned}
\Phi_{2 p}: \mathfrak{O}_{2 p}^{+} & \rightarrow \mathfrak{O}_{2 p}^{+} \\
\mathbf{x}:=(\mathbf{a}, \mathbf{b}) & \rightarrow \mathbf{x}^{+}:=\left(\mathbf{a}^{+}, \mathbf{b}^{+}\right)
\end{aligned}
$$

where $a_{i}^{+}$and $b_{i}^{+}$are given in (5.11), (5.12). Iteration of this map, starting at $\mathbf{x}_{0}$, produces a sequence $\mathbf{x}_{n+1}:=\Phi_{2 p}\left(\mathbf{x}_{n}\right)$ of points in $\mathfrak{O}_{2 p}^{+}$. The rational functions formed with these parameters have integrals that remain constant along this orbit. Numerical studies suggest the existence of a number $L=L\left(\mathbf{x}_{0}\right) \in \mathbb{R}_{+}$such that

$$
\mathbf{x}_{n} \rightarrow\left(\left(\begin{array}{l}
p \\
1
\end{array}\right),\left(\begin{array}{l}
p \\
2
\end{array}\right), \cdots,\left(\begin{array}{c}
p \\
p-1
\end{array}\right) ;\left(\begin{array}{c}
p-1 \\
0
\end{array}\right) L,\left(\begin{array}{c}
p-1 \\
1
\end{array}\right) L, \cdots,\left(\begin{array}{c}
p-1 \\
p-1
\end{array}\right) L\right) .
$$

Thus the integral of the original rational function is $\frac{\pi}{2} \times L$. 


\section{THE SIXTH DEGREE CASE}

We discuss the map $\mathfrak{T}_{2 p}: \mathfrak{E}_{2 p} \rightarrow \mathfrak{E}_{2 p}$ for the case $p=3$. The effect of $\mathfrak{T}_{6}$ on the coefficients $\mathfrak{P}_{6}=\left\{b_{0}, b_{1}, b_{2}, a_{1}, a_{2}\right\}$ is denoted by $\Phi_{6}: \mathfrak{O}_{6}^{+} \rightarrow \mathfrak{D}_{6}^{+}$and is given explicitly by

$$
\begin{aligned}
a_{1} & \rightarrow \frac{9+5 a_{1}+5 a_{2}+a_{1} a_{2}}{\left(a_{1}+a_{2}+2\right)^{4 / 3}}, \\
a_{2} & \rightarrow \frac{a_{1}+a_{2}+6}{\left(a_{1}+a_{2}+2\right)^{2 / 3}}, \\
b_{0} & \rightarrow \frac{b_{0}+b_{1}+b_{2}}{\left(a_{1}+a_{2}+2\right)^{2 / 3}} \\
b_{1} & \rightarrow \frac{b_{0}\left(a_{2}+2\right)+2 b_{1}+b_{2}\left(a_{1}+3\right)}{a_{1}+a_{2}+2}, \\
b_{2} & \rightarrow \frac{b_{0}+b_{2}}{\left(a_{1}+a_{2}+2\right)^{1 / 3}}
\end{aligned}
$$

using Theorem 5.1 The map $\Phi_{6}$ preserves the integral

$$
U_{6}\left(a_{1}, a_{2}, b_{0} ; b_{1}, b_{2}\right):=\int_{0}^{\infty} \frac{b_{0} z^{4}+b_{1} z^{2}+b_{2}}{z^{6}+a_{1} z^{4}+a_{2} x^{2}+1} d z
$$

and the convergence of its iterations has been proved in [3], the main result of which is the following theorem.

Theorem 6.1. Let $\mathbf{x}_{0}:=\left(a_{1}^{0}, a_{2}^{0} ; b_{0}^{0}, b_{1}^{0}, b_{2}^{0}\right) \in \mathbb{R}_{+}^{5}$. Define $\mathbf{x}_{n+1}:=\Phi_{6}\left(\mathbf{x}_{n}\right)$. Then $U_{6}$ is invariant under $\Phi_{6}$. Moreover, the sequence $\left\{\left(a_{1}^{n}, a_{2}^{n}\right)\right\}$ converges to $(3,3)$ and $\left\{\left(b_{0}^{n}, b_{1}^{n}, b_{2}^{n}\right)\right\}$ converges to $(L, 2 L, L)$, where the limit $L$ is a function of the initial data $\mathbf{x}_{0}$. Therefore

$$
\int_{0}^{\infty} \frac{b_{0} z^{4}+b_{1} z^{2}+b_{2}}{z^{6}+a_{1} z^{4}+a_{2} z^{2}+1} d z=L\left(\mathbf{x}_{0}\right) \times \frac{\pi}{2} .
$$

This iteration is similar to Landen's transformation for elliptic integrals that has been employed in [4] in the efficient calculation of $\pi$. Numerical data indicate that the convergence of $\mathbf{x}_{n}$ is quadratic. The proof of convergence is based on the fact that $\Phi_{6}$ cuts the distance from $\left(a_{1}, a_{2}\right)$ to $(3,3)$ by at least half.

A sequence of algebraic curves. The complete characterization of parameters $\left(a_{1}, a_{2}\right)$ in the first quadrant that yield computable rational functions

$$
R(z):=\frac{b_{0} z^{4}+b_{1} z^{2}+b_{2}}{z^{6}+a_{1} z^{4}+a_{2} z^{2}+1}
$$

of degree 6 remains open. The polynomial $z^{6}+a_{1} z^{4}+a_{2} z^{2}+1$ factors when $a_{1}=a_{2}$ so the diagonal $\Delta:=\left\{\left(a_{1}, a_{2}\right) \in \mathbb{R}_{+} \times \mathbb{R}_{+}: a_{1}=a_{2}\right\}$ produces computable functions. In view of the invariance of the class of computable functions under iterations by $\Phi_{6}$, the curves $\mathbb{X}_{n}:=\Phi_{6}^{(-n)}(\Delta)$, with $n \in \mathbb{Z}$, are also computable.

The curve $\mathbb{X}_{1}$ has equation

$$
\left(9+5 a_{1}+5 a_{2}+a_{1} a_{2}\right)^{3}=\left(a_{1}+a_{2}+2\right)^{2}\left(a_{1}+a_{2}+6\right)^{3}
$$

and consists of two branches meeting at the cusp $(3,3)$. In terms of the coordinates $x=a_{1}-3$ and $y=a_{2}-3$ the leading order term is $T_{1}(x, y)=1728(x-y)^{2}$. This 
curve is rational and can be parametrized by

$$
\begin{aligned}
& a_{1}(t)=t^{-2}\left(t^{5}-t^{4}+2 t^{3}-t^{2}+t+1\right), \\
& a_{2}(t)=t^{-3}\left(t^{5}+t^{4}-t^{3}+2 t^{2}-t+1\right) .
\end{aligned}
$$

The rationality of $\mathbb{X}_{n}$ for $n \neq 1$ and its significance for the integration algorithm remains open. The complexity of these curves increases with $n$. For example, the curve $\mathbb{X}_{2}:=\Phi_{3}^{(-2)}(\Delta)$ is of total degree 90 in $x=a_{1}-3$ and $y=a_{2}-3$ with leading term

$$
T_{2}(x, y):=2^{121} 3^{35}(x-y)^{18}\left[-163\left(x^{4}+y^{4}\right)+668 x y\left(x^{2}+y^{2}\right)-1074 x^{2} y^{2}\right] .
$$

The diagonal $\Delta$ can be replaced by a 2 -parameter family of computable curves $\mathbb{X}(c, d)$ that are produced from the factorization of the sextic with $a_{1}=c+d$ and $a_{2}=c d+1 / d$. All the images $\Phi_{6}^{(-n)} \mathbb{X}(c, d)$ with $n \in \mathbb{Z}$ are computable curves. The question of whether these are all the computable parameters remains open.

\section{EXAMPLES}

In this section we present a variety of closed-form evaluations of integrals of rational functions.

Example 1. The integral

$$
\int_{0}^{\infty} \frac{z^{2}}{\left(z^{4}+4 z^{2}+1\right)^{9}} d z=\frac{23698523 \pi}{12230590464 \sqrt{6}}
$$

is computed by Mathematica 3.0 using (3.2) in .01 seconds. The direct calculation took 12.27 seconds and 6.4 extra seconds to simplify the answer.

Example 2. The integral of any even rational function with denominator a power of an even quartic polynomial can be computed directly by using Corollary 3.2 . For example:

$$
\int_{0}^{\infty} \frac{z^{6} d z}{\left(2 z^{4}+2 z^{2}+3\right)^{11}}=\frac{11 \pi(14229567+4937288 \sqrt{6})}{440301256704(1+\sqrt{6})^{21 / 2}} .
$$

Example 3. The case $n=0$ in (3.2) deserves special attention:

$$
N_{0,4}(a ; m)=\frac{\pi}{2^{m+3 / 2}(a+1)^{m+1 / 2}} P_{m}(a)
$$

where

$$
P_{m}(a)=2^{-2 m} \sum_{k=0}^{m} 2^{k}\left(\begin{array}{c}
2 m-2 k \\
m-k
\end{array}\right)\left(\begin{array}{c}
m+k \\
m
\end{array}\right)(a+1)^{k} .
$$

The polynomial $P_{m}(a)$ has been studied in [1] and [2].

Example 4. The case $n=m$ in 3.2 yields

$$
N_{m, 4}(a ; m)=\int_{0}^{\infty} \frac{z^{2 m} d z}{\left(z^{4}+2 a z^{2}+1\right)^{m+1}}=\frac{\pi}{2^{3 m+3 / 2}(1+a)^{m+1 / 2}} \times\left(\begin{array}{c}
2 m \\
m
\end{array}\right) .
$$

The change of variable $z \rightarrow \sqrt{z}$ converts this integral to

$$
N_{m, 4}(a ; m)=\frac{1}{2} \int_{0}^{\infty} \frac{z^{m-1 / 2} d z}{\left(z^{2}+2 a z+1\right)^{m+1}},
$$

which is 8] 3.252.9. 
TABLE 1.

\begin{tabular}{||c|c|c|c|c|c||}
\hline$n$ & $a_{1}^{n}$ & $a_{2}^{n}$ & $b_{0}^{n}$ & $b_{1}^{n}$ & $b_{2}^{n}$ \\
\hline 0 & 1 & 3000 & 45 & 25000 & 1230 \\
1 & .415786 & 14.4465 & 126.233 & 63.2884 & 88.3741 \\
2 & 2.06562 & 3.17262 & 42.2607 & 156.015 & 83.6896 \\
3 & 2.98142 & 3.00338 & 75.3541 & 137.717 & 65.1111 \\
4 & 2.99999 & 3. & 69.6338 & 139.925 & 70.2771 \\
5 & 3. & 3. & 69.9589 & 139.914 & 69.9555 \\
6 & 3. & 3. & 69.9572 & 139.914 & 69.9572 \\
7 & 3. & 3. & 69.9572 & 139.914 & 69.9572 \\
\hline
\end{tabular}

Example 5. A symmetric function of degree 6. The integral

$$
I=\int_{0}^{\infty} \frac{x^{8}}{\left(x^{6}+4 x^{4}+4 x^{2}+1\right)^{5}} d x=\int_{0}^{\infty} \frac{x^{8}}{\left[\left(x^{2}+1\right)\left(x^{4}+3 x^{2}+1\right)\right]^{5}} d x
$$

can be computed by decomposing the integrand into partial fractions as

$$
\begin{array}{r}
-\frac{1}{\left(x^{2}+1\right)^{5}}-\frac{1}{\left(x^{2}+1\right)^{4}}-\frac{6}{\left(x^{2}+1\right)^{3}}-\frac{11}{\left(x^{2}+1\right)^{2}}-\frac{31}{\left(x^{2}+1\right)} \\
+\frac{1}{\left(x^{4}+3 x^{2}+1\right)^{5}}+\frac{2 x^{2}}{\left(x^{4}+3 x^{2}+1\right)^{5}}-\frac{4}{\left(x^{4}+3 x^{2}+1\right)^{4}}-\frac{3 x^{2}}{\left(x^{4}+3 x^{2}+1\right)^{4}} \\
+\frac{12}{\left(x^{4}+3 x^{2}+1\right)^{3}}+\frac{6 x^{2}}{\left(x^{4}+3 x^{2}+1\right)^{3}}-\frac{32}{\left(x^{4}+3 x^{2}+1\right)^{2}}-\frac{14 x^{2}}{\left(x^{4}+3 x^{2}+1\right)^{2}} \\
+\frac{73}{\left(x^{4}+3 x^{2}+1\right)}+\frac{31 x^{2}}{\left(x^{4}+3 x^{2}+1\right)} .
\end{array}
$$

Each of these terms is now computable yielding

$$
I=\frac{1407326 \sqrt{5}-3146875}{160000} \times \pi
$$

Example 6. Nonsymmetric functions of degree 6. In this case we can use the scheme (6.1) to produce numerical approximations to the integral. For example, the evaluation of

$$
\int_{0}^{\infty} \frac{45 z^{4}+25000 z^{2}+1230}{z^{6}+z^{4}+3000 z^{2}+1} d z
$$

is shown in Table 1. Thus $L \sim 69.9572$ and

$$
\int_{0}^{\infty} \frac{45 x^{4}+25000 x^{2}+1230}{x^{6}+x^{4}+3000 x^{2}+1} d x \sim 69.9572 \times \frac{\pi}{2} \cong 109.889
$$

Example 7. Symmetric functions of degree 8. These integrals can be evaluated using Theorem 4.1 For example:

$$
\int_{0}^{\infty} \frac{d z}{\left(z^{8}+5 z^{6}+14 z^{4}+5 z^{2}+1\right)^{4}}=\frac{(14325195794+2815367209 \sqrt{26}) \pi}{14623232(9+2 \sqrt{26})^{7 / 2}} .
$$


Example 8. As in the case of degree 6 we can provide numerical approximations to nonsymmetric integrals of degree 8 . The iteration (6.1) is now replaced by

$$
\begin{aligned}
a_{1}^{n+1} & =\frac{a_{2}^{n}\left(a_{1}^{n}+a_{3}^{n}\right)+4 a_{1}^{n} a_{3}^{n}+10\left(a_{1}^{n}+a_{3}^{n}\right)+8\left(a_{2}^{n}+2\right)}{\left(a_{1}^{n}+a_{2}^{n}+a_{3}^{n}+2\right)^{3 / 2}}, \\
a_{2}^{n+1} & =\frac{a_{1}^{n} a_{3}^{n}+6\left(a_{1}^{n}+a_{3}^{n}\right)+2\left(a_{2}^{n}+10\right)}{a_{1}^{n}+a_{2}^{n}+a_{3}^{3}+2}, \\
a_{3}^{n+1} & =\frac{a_{1}^{n}+a_{3}^{n}+8}{\left(a_{1}^{n}+a_{2}^{n}+a_{3}^{n}+2\right)^{1 / 2}}, \\
b_{0}^{n+1} & =\frac{b_{0}^{n}+b_{1}^{n}+b_{2}^{n}+b_{3}^{n}}{\left(a_{1}^{n}+a_{2}^{n}+a_{3}^{n}+2\right)^{3 / 4}}, \\
b_{1}^{n+1} & =\frac{b_{3}^{n}\left(3 a_{1}^{n}+a_{2}^{n}+6\right)+b_{2}^{n}\left(a_{1}^{n}+4\right)+b_{1}^{n}\left(a_{3}^{n}+4\right)+b_{0}^{n}\left(3 a_{3}^{n}+a_{2}^{n}+6\right)}{\left(a_{1}^{n}+a_{2}^{n}+a_{3}^{n}+2\right)^{5 / 4}}, \\
b_{2}^{n+1} & =\frac{b_{3}^{n}\left(a_{1}^{n}+5\right)+b_{2}^{n}+b_{1}^{n}+b_{0}^{n}\left(a_{3}^{n}+5\right)}{\left(a_{1}^{n}+a_{2}^{n}+a_{3}^{n}+2\right)^{3 / 4}}, \\
b_{3}^{n+1} & =\frac{b_{0}^{n}+b_{3}^{n}}{\left(a_{1}^{n}+a_{2}^{n}+a_{3}^{n}+2\right)^{1 / 4}},
\end{aligned}
$$

with initial conditions $a_{1}^{0}, a_{2}^{0}, a_{3}^{0}, b_{0}^{0}, b_{1}^{0}, b_{2}^{0}, b_{3}^{0}$. Then

$$
U_{8}\left(a_{1}, a_{2}, a_{3}, b_{0}, b_{1}, b_{2}, b_{3}\right):=\int_{0}^{\infty} \frac{b_{0} x^{6}+b_{1} x^{4}+b_{2} x^{2}+b_{3}}{x^{8}+a_{1} x^{6}+a_{2} x^{4}+a_{3} x^{2}+1} d x
$$

is invariant under these transformations.

Note. Numerical calculations show that $\left(a_{1}^{n}, a_{2}^{n}, a_{3}^{n}\right) \rightarrow(4,6,4)$ and that $\left(b_{0}^{n}, b_{1}^{n}, b_{2}^{n}, b_{3}^{n}\right) \rightarrow(1,3,3,1) L$ for some $L$ depending upon the initial conditions.

Example 9. A symmetric function of degree 12. We use Theorem 2.1 to evaluate

$$
I:=\int_{0}^{\infty} \frac{z^{18} d z}{\left(z^{12}+14 z^{10}+15 z^{8}+4 z^{6}+15 z^{4}+14 z^{2}+1\right)^{3}}
$$

as

$$
I=\frac{25 \pi(25 \sqrt{56}-54)}{301989888} .
$$

Here $p=3, n=9$, and $m=2$, so $n>(m+1) p-1$ and we need to apply the transformation $z \rightarrow 1 / z$ to reduce the value of $n$. Indeed, we have

$$
I=\int_{0}^{\infty} \frac{z^{16} d z}{\left(z^{12}+14 z^{10}+15 z^{8}+4 z^{6}+15 z^{4}+14 z^{2}+1\right)^{3}},
$$

and Theorem 2.1 now yields

$$
I=2^{-17} \int_{0}^{\infty} \frac{z^{16} d z}{131072\left(1+z^{2}\right)^{3}\left(1+4 z^{2}+z^{4}\right)^{3}} .
$$

The new integrand is expanded in partial fractions in the variable $t=z^{2}$ to produce (7.4).

Example 10. We use Theorem 2.1 to evaluate

$$
I:=\int_{0}^{\infty} \frac{z^{10} d z}{Q^{2}(z)}
$$


where

$$
\begin{aligned}
Q(z)= & z^{20}+6 z^{18}+93 z^{16}-24 z^{14}+162 z^{12} \\
& +548 z^{10}+162 z^{8}-24 z^{6}+93 z^{4}+6 z^{2}+1 .
\end{aligned}
$$

The factorization

$$
Q(z)=\left(1+z^{2}\right)^{2} T(z) T(-z)
$$

with

$$
T(z)=z^{8}-2 z^{7}+4 z^{6}+14 z^{5}+6 z^{4}-14 z^{3}+4 z^{2}+2 z+1
$$

leads to a partial fraction expansion containing the term

$$
\frac{72-501 z+1994 z^{2}-2617 z^{3}+1228 z^{4}-43 z^{5}+34 z^{6}-55 z^{7}}{8388608\left(1-2 z+4 z^{2}+14 z^{3}+6 z^{4}-14 z^{5}+4 z^{6}+2 z^{7}+z^{8}\right)^{2}}
$$

which we were unable to integrate; furthemore, the roots of $T(z)=0$ cannot be evaluated by radicals. The procedure described in Theorem 2.1, however, shows that

$$
I=\int_{0}^{\infty} \frac{z^{10}\left(4+z^{2}\right)\left(z^{6}+36 z^{4}+96 z^{2}+64\right) d z}{524288\left(z^{2}+1\right)^{2}\left(z^{8}+3 z^{6}+8 z^{4}+3 z^{2}+1\right)^{2}},
$$

the integrand of which can be expanded to yield

$$
\begin{aligned}
I= & -\frac{9}{8388608} \int_{0}^{\infty} \frac{d z}{\left(z^{2}+1\right)^{2}}-\frac{75}{8388608} \int_{0}^{\infty} \frac{d z}{z^{2}+1} \\
& +\int_{0}^{\infty} \frac{1921 z^{6}+10815 z^{4}+4111 z^{2}+1462}{2097152\left(z^{8}+3 z^{6}+8 z^{4}+3 z^{2}+1\right)^{2}} d z \\
& +\int_{0}^{\infty} \frac{91 z^{6}+719 z^{4}+1259 z^{2}-5764}{8388608\left(z^{8}+3 z^{6}+8 z^{4}+3 z^{2}+1\right)} d z .
\end{aligned}
$$

Every piece is now computable, with the final result

$$
I=\frac{(6480-509 \sqrt{15}) \pi}{24159191040} .
$$

Example 11. The symmetric functions of degree 16 have denominator

$$
\begin{aligned}
D_{4}\left(d_{1}, d_{2}, d_{3}, d_{4} ; z\right)= & z^{16}+d_{4} z^{14}+d_{3} z^{12}+d_{2} z^{10} \\
& +2 d_{1} z^{8}+d_{2} z^{6}+d_{3} z^{4}+d_{4} z^{2}+1,
\end{aligned}
$$

the integral of which is computed in terms of

$$
\begin{aligned}
E_{4}\left(d_{1}, d_{2}, d_{3}, d_{4} ; z\right)= & \left(1+d_{1}+d_{2}+d_{3}+d_{4}\right) z^{8}+2\left(16+d_{2}+4 d_{3}+9 d_{4}\right) z^{6} \\
& +8\left(20+d_{3}+6 d_{4}\right) z^{4}+32\left(8+d_{4}\right) z^{2}+128 .
\end{aligned}
$$

This new integral is symmetric provided

$$
\left[\begin{array}{l}
d_{1} \\
d_{2}
\end{array}\right]=\left[\begin{array}{c}
15 \\
112
\end{array}\right]+\left[\begin{array}{c}
3 \\
-4
\end{array}\right] d_{3}+\left[\begin{array}{c}
-8 \\
7
\end{array}\right] d_{4}
$$

Introduce the new parameters

$$
e_{j}=d_{j}-\left(\begin{array}{c}
8 \\
5-j
\end{array}\right) \text { for } 2 \leq j \leq 4 \text { and } e_{1}=d_{1}-\frac{1}{2}\left(\begin{array}{l}
8 \\
4
\end{array}\right) .
$$


Then (7.5) yields

$$
\left[\begin{array}{l}
e_{1} \\
e_{2}
\end{array}\right]=\left[\begin{array}{c}
3 \\
-4
\end{array}\right] e_{3}+\left[\begin{array}{c}
-8 \\
7
\end{array}\right] e_{4}
$$

Thus, if the original denominator has the form

$$
\begin{aligned}
D_{4}(z)= & \left(z^{16}+1\right)+d_{4}\left(z^{14}+z^{2}\right)+d_{3}\left(z^{12}+z^{4}\right)+\left(112-4 d_{3}+7 d_{4}\right)\left(z^{10}+z^{6}\right) \\
& +2\left(15+3 d_{3}-8 d_{4}\right) z^{8},
\end{aligned}
$$

the integral

$$
\int_{0}^{\infty} \frac{P(z)}{\left(D_{4}(z)\right)^{m+1}} d z
$$

is reduced to an integral with symmetric denominator of degree 8 and these are computable. We can thus evaluate a 2-parameter family of symmetric integrals of degree 16.

For example, take $d_{3}=d_{4}=1$ to obtain

$$
R_{1}(z)=\frac{z^{4}}{\left(z^{16}+z^{14}+z^{12}+115 z^{10}+20 z^{8}+115 z^{6}+z^{4}+z^{2}+1\right)^{2}}
$$

The main theorem yields

$$
R_{2}(z)=\frac{1024 z^{4}+2304 z^{6}+1792 z^{8}+560 z^{10}+60 z^{12}+z^{14}}{2^{7}\left(16 z^{8}+36 z^{6}+27 z^{4}+36 z^{2}+16\right)^{2}}
$$

so that

$$
\int_{0}^{\infty} R_{1}(z) d z=\int_{0}^{\infty} R_{2}(z) d z .
$$

Letting $f[n]:=N_{n, 8}[1, n, 27 / 32,9 / 4]$ we obtain

$$
\int_{0}^{\infty} R_{1}(z) d z=2^{-15}(f[0]+60 f[1]+1584 f[2]+4096 f[3])
$$

and conclude that

$$
\begin{gathered}
\int_{0}^{\infty} \frac{z^{4} d z}{\left(z^{16}+z^{14}+z^{12}+115 z^{10}+20 z^{8}+115 z^{6}+z^{4}+z^{2}+1\right)^{2}} \\
=\frac{(149288517+12947003 \sqrt{131}) \pi}{1124663296 \sqrt{54925+4798 \sqrt{131}}} .
\end{gathered}
$$

Example 12. We classify the symmetric denominators of degree 32 that yield computable integrals. These functions depend on 8 parameters

$$
D_{8}\left(d_{1}, \cdots, d_{8} ; z\right)=\sum_{k=0}^{8} d_{9-k}\left(z^{2 k}+z^{2(16-k)}\right)
$$

and the main theorem expresses the integral in terms of $E_{8}$. The conditions for $E_{8}$ to be symmetric yield

$$
\begin{aligned}
& d_{1}=-3441+35 d_{5}+64 d_{6}-312 d_{7}-3264 d_{8}, \\
& d_{2}=34720-56 d_{5}-110 d_{6}+560 d_{7}+4565 d_{8}, \\
& d_{3}=-3472+28 d_{5}+64 d_{6}-329 d_{7}-2240 d_{8}, \\
& d_{4}=4960-8 d_{5}-19 d_{6}+80 d_{7}+938 d_{8},
\end{aligned}
$$


and the symmetric $E_{8}$ is

$$
\begin{aligned}
E_{8}\left(d_{5}, d_{6}, d_{7}, d_{8} ; z\right)= & 32768\left(1+z^{16}\right)+\left(131072+8192 d_{8}\right)\left(z^{2}+z^{14}\right) \\
& +\left(212992+2048 d_{7}+28672 d_{8}\right)\left(z^{4}+z^{12}\right) \\
& +\left(180224+512 d_{6}+6144 d_{7}+39424 d_{8}\right)\left(z^{6}+z^{10}\right) \\
& +\left(84480+128 d_{5}+1280 d_{6}+6912 d_{7}+26880 d_{8}\right) z^{8} .
\end{aligned}
$$

The symmetry of $E_{8}$ now determines $d_{5}, d_{6}$ in terms of $d_{7}, d_{8}$ and we obtain

$$
\left[\begin{array}{l}
d_{1} \\
d_{2} \\
d_{3} \\
d_{4} \\
d_{5} \\
d_{6}
\end{array}\right]=-31\left[\begin{array}{c}
63475 \\
-100800 \\
47936 \\
-13664 \\
2220 \\
-224
\end{array}\right]+\left[\begin{array}{c}
9166 \\
-14392 \\
6895 \\
-1964 \\
322 \\
-28
\end{array}\right] d_{7}+\left[\begin{array}{c}
54640 \\
-86645 \\
41664 \\
-11471 \\
2000 \\
-189
\end{array}\right] d_{8} .
$$

The function $\left(z^{2}+1\right)^{16}$ is a symmetric polynomial of degree 32 and yields a particular solution to (7.10).

As before let

$$
e_{j}=d_{j}-\left(\begin{array}{c}
16 \\
9-j
\end{array}\right) \text { for } 2 \leq j \leq 8 \text { and } e_{1}=d_{1}-\frac{1}{2}\left(\begin{array}{c}
16 \\
8
\end{array}\right) .
$$

Then

$$
\left[\begin{array}{l}
e_{1} \\
e_{2} \\
e_{3} \\
e_{4} \\
e_{5} \\
e_{6}
\end{array}\right]=\left[\begin{array}{c}
9166 \\
-14392 \\
6895 \\
-1964 \\
322 \\
-28
\end{array}\right] e_{7}+\left[\begin{array}{c}
54640 \\
-86645 \\
41664 \\
-11471 \\
2000 \\
-189
\end{array}\right] e_{8}
$$

and as in the case of degree 16 we can compute a 2-parameter family of symmetric integrals of degree 32 .

\section{Appendix A. Two Binomial sums}

The closed-form evaluation of sums involving binomial coefficients can be obtained by traditional analytical techniques or by using the powerful WZ-method as described in [16. We discuss two sums used to simplify expressions in later sections, presenting one proof in each style.

Lemma A.1. Let $k, N$ be positive integers with $k \leq N$. Then

$$
\sum_{j=0}^{N-k}\left(\begin{array}{c}
2 N+1 \\
2 j
\end{array}\right)\left(\begin{array}{c}
N-j \\
k
\end{array}\right)=\left(\begin{array}{c}
2 N-k \\
k
\end{array}\right) 4^{N-k}
$$


Proof. Multiply the left-hand side of (A.1) by $x^{k}$ and sum over $k$ to produce

$$
\begin{aligned}
\sum_{k=0}^{N} \sum_{j=0}^{N-k}\left(\begin{array}{c}
2 N+1 \\
2 j
\end{array}\right)\left(\begin{array}{c}
N-j \\
k
\end{array}\right) x^{k} & =\sum_{j=0}^{N}\left(\begin{array}{c}
2 N+1 \\
2 j
\end{array}\right) \sum_{k=0}^{N-j}\left(\begin{array}{c}
N-j \\
k
\end{array}\right) x^{k} \\
& =\sum_{j=0}^{N}\left(\begin{array}{c}
2 N+1 \\
2 j
\end{array}\right)(x+1)^{N-j} \\
& =\frac{(1+\sqrt{x+1})^{2 N+1}-(1-\sqrt{x+1})^{2 N+1}}{2 \sqrt{x+1}} \\
& =\sum_{k=0}^{N}\left(\begin{array}{c}
2 N-k \\
k
\end{array}\right) 4^{N-k} x^{k} .
\end{aligned}
$$

In order to justify the last step we start with the well-known result

$$
\frac{1}{\sqrt{1-4 y}}\left(\frac{1-\sqrt{1-4 y}}{2 y}\right)^{i}=\sum_{k}\left(\begin{array}{c}
2 k+i \\
k
\end{array}\right) y^{k}
$$

(see WILF [19], page 54). Letting $x=-4 y$ and $i=2 N+1$ in (A.2) gives

$$
\frac{(1-\sqrt{x+1})^{2 N+1}}{2 \sqrt{x+1}}=\sum_{k=0}^{\infty}(-1)^{k+1}\left(\begin{array}{c}
2 N+1+2 k \\
k
\end{array}\right) 4^{-(N+1+k)} x^{2 N+1+k} ;
$$

similarly $x=-4 y$ and $i=-2 N-1$ yields

$$
\frac{(1+\sqrt{x+1})^{2 N+1}}{2 \sqrt{x+1}}=\sum_{k=2 N+1}^{\infty}(-1)^{k+1}\left(\begin{array}{c}
2 N+1+2 k \\
k
\end{array}\right) 4^{-(N+1+k)} x^{2 N+1+k} .
$$

Thus

$$
\begin{aligned}
\frac{(1+\sqrt{x+1})^{2 N+1}-(1-\sqrt{x+1})^{2 N+1}}{2 \sqrt{x+1}} & =\sum_{k=0}^{2 N}(-1)^{k}\left(\begin{array}{c}
-(2 N+1-2 k) \\
k
\end{array}\right) 4^{N-k} x^{k} \\
& =\sum_{k=0}^{N}\left(\begin{array}{c}
2 N-k \\
k
\end{array}\right) 4^{N-k} x^{k} .
\end{aligned}
$$

Lemma A.2. Let $N \in \mathbb{N}$. Then

$$
\sum_{j=0}^{N}\left(\begin{array}{c}
2 N+1 \\
2 j
\end{array}\right)\left(1+z^{2}\right)^{N-j}=\sum_{j=0}^{N}\left(\begin{array}{c}
N+j \\
2 j
\end{array}\right) 4^{j} z^{2(N-j)} .
$$

Proof. The coefficient of $z^{2 k}$ on the left-hand side is

$$
\sum_{j=0}^{N-k}\left(\begin{array}{c}
2 N+1 \\
2 j
\end{array}\right)\left(\begin{array}{c}
N-j \\
k
\end{array}\right)
$$

and the corresponding coefficient on the right-hand side is $\left(\begin{array}{c}2 N-k \\ k\end{array}\right) 4^{N-k}$. The result then follows from Lemma A.1.

Lemma A.3. Let $k, N \in \mathbb{N}$ with $k \leq N$. Then

$$
\sum_{j=0}^{k}\left(\begin{array}{c}
2 N \\
2 j
\end{array}\right)\left(\begin{array}{c}
N-j \\
N-k
\end{array}\right)=\frac{2^{2 k-1} N}{k}\left(\begin{array}{c}
k+N-1 \\
N-k
\end{array}\right) .
$$


Proof. This lemma could be proven in the same style as Lemma A.1. Instead we use the WZ-method as explained in [16]. Indeed, let

$$
F(k ; j)=\frac{k\left(\begin{array}{c}
2 N \\
2 j
\end{array}\right)\left(\begin{array}{c}
N-j \\
N-k
\end{array}\right)}{N 2^{2 k-1}\left(\begin{array}{c}
k+N-1 \\
N-k
\end{array}\right)},
$$

and define, with the package EKHAD, the function

$$
G(k ; j)=F(k ; j) \times \frac{j(2 j-1)}{2(N+k)(k-j+1)} .
$$

Then $F(k ; j)-F(k+1 ; j)=G(k ; j+1)-G(k ; j)$, and summing over $j$ we see that the sum of $F(k ; j)$ over $j$ is independent of $k$. The case $k=N$ produces 1 as the common value.

Lemma A.4. Let $p \in \mathbb{N}, d_{1}, d_{2}, \cdots, d_{p}$ be parameters, and define $d_{p+1}:=1$. Then

$$
\begin{aligned}
& \sum_{k=0}^{p} d_{p+1-k} z^{2 k} \sum_{j=0}^{p-k}\left(\begin{array}{c}
2 p-2 k \\
2 j
\end{array}\right)\left(1+z^{2}\right)^{p-k-j} \\
& \quad=\left(\sum_{j=1}^{p+1} d_{j}\right) z^{2 p}+\sum_{i=1}^{p} 2^{2 i-1} z^{2(p-i)}\left(\sum_{j=1}^{p+1-i} \frac{j+i-1}{i}\left(\begin{array}{c}
j+2 i-2 \\
j-1
\end{array}\right) d_{j+i}\right)
\end{aligned}
$$

Proof. For fixed $0 \leq i \leq p-1$ the coefficient of $z^{2 i}$ on the right-hand side of (A.3) is

$$
[\mathrm{RHS}](2 i)=\frac{2^{2(p-i)-1}}{p-i} \sum_{r=p-i+1}^{p+1}(r-1)\left(\begin{array}{c}
r+p-i-2 \\
r-p+i-1
\end{array}\right) d_{r},
$$

and for $i=p$ we have [RHS] $(2 p)=1+\sum_{j=1}^{p} d_{j}$. Similarly, for the left-hand side of (A.3),

$$
[\text { LHS }](2 i)=\sum_{r=p+1-i}^{p+1} d_{r}\left(\sum_{j=0}^{p-i}\left(\begin{array}{c}
2 r-2 \\
2 j
\end{array}\right)\left(\begin{array}{c}
r-j-1 \\
r-j-1+i
\end{array}\right)\right) .
$$

It is easy to check that the coefficients of $z^{2 p}$ match. It suffices to show that for each $i$ such that $0 \leq i \leq p-1$ and for each $r$ such that $p+1-i \leq r \leq p+1$ we have

$$
\sum_{j=0}^{p-i}\left(\begin{array}{c}
2 r-2 \\
2 j
\end{array}\right)\left(\begin{array}{c}
r-1-j \\
r-1-p+i
\end{array}\right)=\frac{2^{2(p-i)-1}}{p-i}(r-1)\left(\begin{array}{c}
r+p-i-2 \\
r-p+i-1
\end{array}\right) .
$$

This follows from Lemma A.1 with $k=p-i$ and $N=r-1$.

The suggestions of the referees and the editor are gratefully acknowledged.

\section{REFERENCES}

[1] BOROS, G. and MOLL, V.: A criterion for unimodality. Elec. Jour. of Combinatorics, 6 (1999), \#R10. MR 99k:05017

[2] BOROS, G. and MOLL, V.: An integral hidden in Gradshteyn and Ryzhik. Jour. Comp. Appl. Math. 106, 361-368, 1999. MR 2000c:33024

[3] BOROS, G. and MOLL, V.: A rational Landen transformation. Contemporary Mathematics 251, 83-91, 2000. MR 2001h:33029 
[4] BORWEIN, J. and BORWEIN, P.: Pi and the AGM. Canadian Mathematical Society. WileyInterscience Publication. MR 89a:11134, MR 99h:11147

[5] BRONSTEIN, M.: Symbolic Integration I. Transcendental functions. Algorithms and Computation in Mathematics, 1. Springer-Verlag, 1997. MR 98a:68104

[6] GEDDES, K., CZAPOR, S.R. and LABAHN, G.: Algorithms for Computer Algebra. Kluwer, Dordrecht. The Netherlands, 1992. MR 96a:68049

[7] GAUSS, K.F.: Arithmetische Geometrisches Mittel, 1799. In Werke, 3, 361-432. Konigliche Gesellschaft der Wissenschaft, Gottingen. Reprinted by Olms, Hildescheim, 1981.

[8] GRADSHTEYN, I.S. and RYZHIK, I.M.: Table of Integrals, Series and Products. Fifth Edition, ed. Alan Jeffrey. Academic Press, 1994. MR 94g:00008

[9] HARDY, G.H.: The Integration of Functions of a Single Variable. Cambridge Tracts in Mathematics and Mathematical Physics, 2, Second Edition, Cambridge University Press, 1958. MR 50:2417

[10] HERMitE, C.: Sur l'integration des fractions rationelles. Nouvelles Annales de Mathematiques ( $2^{\mathrm{eme}}$ serie) $\mathbf{1 1}, 145-148,1872$.

[11] HOROWITZ, E.: Algorithms for partial fraction decomposition and rational function integration. Proc. of SYMSAM'71, ACM Press, 441-457, 1971.

[12] LAZARD, D. and RIOBOO, R.: Integration of Rational Functions: Rational Computation of the Logarithmic Part. Journal of Symbolic Computation 9, 113-116, 1990. MR 91h:68091

[13] MCKEAN, H. and MOLL, V.: Elliptic Curves: Function Theory, Geometry, Arithmetic. Cambridge University Press, 1997. MR 98g:14032

[14] NEWMAN, D.: A simplified version of the fast algorithm of Brent and Salamin. Math. Comp. 44, 207-210, 1985. MR 86e:65030

[15] OSTROGRADSKY, M.W.: De l'integration des fractions rationelles. Bulletin de la Classe Physico-Mathematiques de l'Academie Imperieriale des Sciences de St. Petersbourgh, IV, 145-167, 286-300. 1845.

[16] PetkovseK, M., WILf, H.S. and ZeIlberGeR, D.: $A=B$. A. K. Peters, Wellesley, Massachusetts. 1996. MR 97j:05001

[17] ROTHSTEIN, M.: A new algorithm for the integration of Exponential and Logarithmic Functions, Proc. of the 1977 MACSYMA Users Conference, NASA Pub., CP-2012, 263-274.

[18] TRAGER, B.M.: Algebraic factoring and rational function integration. Proc. SYMSAC 76, 219-226.

[19] WILF, H.S.: generatingfunctionology. Academic Press, 1990. MR 91g:05008

Department of Mathematics, Xavier University, New Orleans, Louisiana 70125

E-mail address: gboros@xula.math.edu

Department of Mathematics, Tulane University, New Orleans, Louisiana 70118

E-mail address: vhm@math.tulane.edu 